Slavica

bruxellensia

\section{Slavica bruxellensia}

Revue polyphonique de littérature, culture et histoire

slaves

$3 \mid 2009$

Pensée des hommes

\title{
Boris Mouravieff et l'ésotérisme chrétien
}

\section{Olivier Santamaria}

\section{OpenEdition \\ Journals}

Édition électronique

URL : http://journals.openedition.org/slavica/273

DOI : $10.4000 /$ slavica. 273

ISSN : 2034-6395

Éditeur

Université libre de Bruxelles - ULB

Édition imprimée

Pagination : 48-60

ISSN : 2031-7654

\section{Référence électronique}

Olivier Santamaria, «Boris Mouravieff et l'ésotérisme chrétien », Slavica bruxellensia [En ligne], 3 | 2009, mis en ligne le 15 juin 2009, consulté le 30 avril 2019. URL : http://journals.openedition.org/ slavica/273 ; DOI : 10.4000/slavica.273

Ce document a été généré automatiquement le 30 avril 2019

\section{(c) (i) $\odot$}

Les contenus de Slavica bruxellensia sont mis à disposition selon les termes de la Licence Creative Commons Attribution - Pas d'Utilisation Commerciale - Pas de Modification 3.0 France. 


\title{
Boris Mouravieff et l'ésotérisme chrétien
}

\author{
Olivier Santamaria
}

\section{NOTE DE L'ÉDITEUR}

Olivier Santamaria propose une critique des Écrits sur Ouspensky, Gurdjieff et sur la Tradition ésotérique chrétienne de Boris Mouravieff dans la rubrique « Recensions »

1 Historien, philosophe, ésotériste et maître spirituel, Boris Mouravieff fut avant tout un homme inclassable. Professeur d'histoire à l'université de Genève, il quitte ses fonctions officielles pour se consacrer à l'enseignement ésotérique. Russe expatrié en Occident, il n'affiche pas l'exclusivisme de certains orthodoxes à l'égard des autres confessions chrétiennes. Slavophile, amoureux de la vieille Russie et de tout l'Orient chrétien, il ne réduit pourtant pas sa pensée religieuse à la Tradition chrétienne seule puisque, pour lui, la véritable Tradition n'est ni Russe ni Grecque et, plus largement, ni d'Orient ni d'Occident : elle est universelle. Certains de ses commentateurs ont voulu faire de lui un orthodoxe ; or, s'il est indéniable que Mouravieff a effectivement opté pour la religion de ses ancêtres, il est néanmoins toujours resté distant de toute Église institutionnelle. Pour lui, en effet, la foi du simple fidèle, si elle suffit au salut individuel, ne procure pourtant pas la pleine connaissance - Gnôsis. Seule la connaissance, entendue comme une connaissance initiatique, ésotérique, peut faire parvenir le fidèle à la seconde Naissance, aux portes du Royaume de Dieu, et lui permettre de guider l'humanité, par-delà la crise qui la menace, vers une destinée heureuse. C'est à cette connaissance, transmise jusqu'à lui par la Tradition ésotérique de l'orthodoxie orientale, que Mouravieff consacrera la plus grande part de ses œuvres et de son énergie.

2 Pour lui, la « Tradition ésotérique », bien qu'universelle par nature, se révèle d'une façon différenciée en fonction des époques et de la mentalité des peuples auxquels elle s'adresse. Face à un public essentiellement Européen, il est naturel que Mouravieff ait 
présenté son enseignement sous une forme chrétienne car, chrétien ou non, croyant ou athée, l'homme européen est façonné par vingt siècles de culture chrétienne. Par ailleurs, contrairement aux autres religions qui, bien qu'issues de la Tradition unique, ne s'adressent toujours qu'à une fraction de l'humanité, le christianisme a affirmé dès ses origines un caractère œcuménique et universaliste. Pour ces deux raisons, l'enseignement de Mouravieff est donc " profondément chrétien »'.

Quant à «l'ésotérisme ", il signifie pour lui principalement une méthode d'interprétation et de compréhension approfondie des textes sacrés, associée à des méthodes de perfectionnement de la "Personne». La doctrine ésotérique est comprise comme un équivalent approximatif de "Gnose » ou de "Connaissance », au sens d'une Connaissance vivante, qui procure des niveaux de conscience élevés, et qui transforme le sujet luimême, comme lors d'un processus initiatique ${ }^{2}$. Boris Mouravieff a d'ailleurs voulu organiser son système selon une structure de mouvement initiatique : l'étudiant prend connaissance en premier lieu de l'aspect théorique et relativement extérieur de la doctrine ; ensuite il avance dans la connaissance de celle-ci par différents paliers; enfin il est initié à la pratique, qui requiert une intériorisation, un approfondissement et une réalisation concrète, ce qui équivaut à une véritable seconde Naissance.

\section{Quelques éléments biographiques}

Boris Mouravieff est né à Kronstadt le 8 mars 1890 et décédé à Genève en 1969, à l'âge de 79 ans. Située sur l'île Kotlin (Golfe de Finlande) en Russie, Kronstadt était rattachée administrativement à Saint-Pétersbourg et servait principalement de base navale. La ville de Kronstadt est restée célèbre pour avoir été à l'avant-garde des révolutions de 1905 et de 1917, ainsi que le siège de la « Révolte » éponyme, qui opposa les marins anarchistes et révolutionnaires au pouvoir bolchevique en 1921.

5 Fils du comte Pjotr Petrovič Murav'ev, amiral de la Flotte russe et dernier secrétaire d'État à la Marine de Guerre impériale, Boris était destiné à une carrière militaire ou politique. À l'abdication du tsar en mars 1917, alors qu'il n'a que 27 ans, il est promu successivement capitaine de frégate puis chef de cabinet naval du ministre socialiste révolutionnaire Alexandr Fjodorovič Kerenskij dans le premier gouvernement provisoire (sous la direction du prince Georgij L'vov). Il devient ensuite chef d'état-major adjoint de la flotte de la mer Noire, nommé par le même Kerenskij, qui était alors chef du gouvernement russe, jusqu'au renversement de ce dernier lors du coup d'état du 7 novembre 1917 qui marque le début de la « Grande Révolution socialiste d'Octobre » et les premières heures du régime bolchevique.

6 Ces événements marquent la fin des activités politiques et militaires de Boris Mouravieff. Ses écrits et sa pensée demeureront néanmoins toujours empreints d'un intérêt réel pour la question politique ou métapolitique.

7 L'influence spirituelle de son grand-oncle sera elle aussi - et peut-être même surtout déterminante. Andrej Nikolaevič Murav'ev, homme de lettres issu de la noblesse russe, s'était attaché, lors de nombreux voyages en Orient, à retrouver une série de manuscrits anciens touchant aux fondements de la tradition orthodoxe. Il sera l'auteur d'une Histoire de l'Église de Russie ${ }^{3}$ ainsi que le fondateur du skite (groupe d'ermites vivant sous l'autorité 
d'un monastère) russe Saint André au Mont Athos. Boris Mouravieff évoque son souvenir et son influence en ces termes :

Personnellement, je m'occupe de la Tradition en question depuis ma jeunesse ; j'en étais favorisé aussi par certaines indications dues à la tradition de ma famille, issue d'André Mouravieff (mort en 1874), Chambellan à la Cour impériale, membre du Saint-Synode, fondateur du monastère de Saint-André au Mont-Athos. C'était un grand voyageur. Il parcourut l'Égypte, l'Asie Antérieure, l'Arménie, la Transcaucasienne, le Kurdistan et poussa jusqu'à la Perse, et au delà, les recherches des détails de cette Tradition sublime et des manuscrits des premiers siècles de notre ère. ${ }^{4}$

Boris Mouravieff poursuivra la voie tracée par son grand-oncle, en s'attachant toute sa vie à retrouver puis à enseigner ce qu'il appellera lui-même la " Tradition ésotérique de l'orthodoxie orientale » ou plus simplement la « Tradition ».

En 1920 il se rend à Constantinople pour y suivre des conférences données par Pjotr Dem'janovič Uspenskij [Ouspensky] (Moscou, 1878 - Londres, 1947), philosophe et maître spirituel d'origine russe. Ce dernier lui fera rencontrer Georgij Ivanovič Gjurdžiev [Gurdjieff] (Alexandropol [Russie], 1866 - Paris, 1949) qui l'introduira auprès de plusieurs personnes en France, dans son Prieuré de Fontainebleau ainsi qu'à Paris. Dans ses articles et livres touchant à l'ésotérisme, Mouravieff prendra certaines distances par rapport à ces auteurs, dont il juge les enseignements fragmentaires. La controverse autour des Fragments d'un enseignement inconnu ${ }^{5}$, ouvrage dans lequel Uspenskij présentait le « système » de Gjurdžiev, est révélatrice d'une tension qui existait depuis le début entre les trois hommes'.

10 Arrivé en France en 1924, Mouravieff s'installe à Bordeaux et y rencontre une Russe, Larisa Basov. Elle deviendra sa femme en 1936, année où ils s'installent à Paris. Jusqu'en 1941, il travaille comme ingénieur consultant dans diverses compagnies pétrolières, tout en se consacrant à l'étude et à ses écrits. Il rencontre aussi fréquemment Gjurdžiev et quelques-uns de ses disciples au Café de la Paix sur les Grands Boulevards de Paris. Les débats et controverses qui y prennent place sont pour lui l'occasion d'affiner son propre point de vue. Pendant la Deuxième Guerre mondiale, en 1944, refusant de collaborer avec les Allemands, il est arrêté par la Gestapo, mais parvient à s'échapper en Suisse avec sa famille. Alors âgé de 55 ans, il entreprend un cursus universitaire à l'Institut des Hautes Études Internationales à Genève, où il défend en 1951 une thèse sur l'Alliance Russoturque pendant la période napoléonienne. Quatre années plus tard, il devient privatdozent (enseignant en attente d'une chaire universitaire) à l'Université de Genève après avoir présenté une leçon sur «La politique de Pierre le Grand dans la question d'Orient ». Cette fonction universitaire couronne ses recherches d'historien qui s'étaient déjà vues concrétisées par la publication de plusieurs ouvrages, notamment Le Testament de Pierre le Grand, légende et réalité ${ }^{7}$ et La Monarchie Russe ${ }^{8}$, ainsi que plusieurs articles9.

\section{L'ésotérisme, une philosophie de l'histoire}

11 Outre son intérêt pour l'histoire, Mouravieff s'intéresse à la spiritualité orthodoxe, ce qui lui vaut de donner un cours à l'université de Genève qu'il intitule «Introduction à la philosophie ésotérique d'après la tradition ésotérique de l'Orthodoxie orientale ». Ce cours, qui réunit régulièrement une vingtaine d'élèves, sert de base à la publication en 1961 d'un premier volume de son œuvre principale, Gnôsis. Études et commentaires sur la tradition ésotérique de l'orthodoxie orientale, volume auquel seront adjoints en 1962 et 1965 
un deuxième puis un troisième tome. Respectivement sous-titrés "cycle exotérique ", «cycle mésotérique» et "cycle ésotérique», Mouravieff y défend l'idée qu'une progression par degrés dans l'enseignement de la doctrine est indispensable pour accéder au niveau ésotérique. Il s'agit selon lui de passer des aspects externes (la profession de foi, les dogmes, qui sont vrais mais d'une façon relative car ils ne sont que l'expression formalisée d'un mystère) aux aspects internes de la doctrine, cachés au regard du profane, et qui constituent la véritable connaissance (gnôsis, en grec).

Parallèlement à ses écrits, Mouravieff anime entre 1961 et 1967 un séminaire de recherches ésotériques destiné à approfondir l'enseignement compilé dans Gnôsis. Le Centre d'Études Chrétiennes Ésotériques (C.E.C.E.) se proposait en effet de développer et de mettre en pratique la doctrine ésotérique, et plus largement de contribuer à l'émergence de «l'Homme Nouveau », expression qui fait référence au mot de l'Apôtre Paul :

Il vous faut abandonner votre premier genre de vie et dépouiller le vieil homme, qui va se corrompant au fil des convoitises décevantes, pour vous renouveler par une transformation spirituelle de votre jugement et revêtir l'Homme Nouveau, qui a été créé selon Dieu, dans la justice et la sainteté de la vérité. ${ }^{10}$

Pour faire émerger cet Homme Nouveau, celui dont l'intelligence est renouvelée par le don de l'Esprit, Mouravieff pense qu'il est nécessaire de rendre publique la doctrine ésotérique. Une nouvelle élite devrait alors se mettre en place, consciente de la marche du monde et du rôle qu'elle doit y jouer, c'est-à-dire œuvrer à faire progresser l'humanité dans une nouvelle phase, à tous égards décisive, de son évolution.

Mouravieff combine en quelque sorte ses deux domaines de prédilection (l'histoire et la spiritualité) dans une conception théologique de l'histoire, à mi-chemin entre une conception cyclique et une conception strictement linéaire de l'évolution humaine. Pour lui, le temps peut être divisé en trois ${ }^{11}$ phases qualitativement distinctes :

Selon la Tradition, l'évolution humaine, après une longue période préhistorique, se poursuit dans une succession de trois cycles: Cycle du Père, que l'histoire ne connaît qu'incomplètement; Cycle du Fils, qui tend à sa fin ; enfin, Cycle du SaintEsprit, auquel nous parvenons actuellement. ${ }^{12}$

Cette idée n'est pas neuve dans la pensée chrétienne. Le moine calabrais Joachim de Flore (1130 - 1202) avait, en effet, déjà exprimé une conception de l'histoire expliquant l'avancement de l'humanité comme un dévoilement progressif des trois personnes de la sainte Trinité : l'âge du Père, associé à la Loi, l'âge du Fils, associé à la Grâce, à la Foi et à l'enseignement dogmatique de l'Église charnelle, l'âge de l'Esprit saint, associé à la surabondance de la Grâce, à la plénitude de la Connaissance et de l'Amour d'une Église purement spirituelle. La postérité spirituelle de cette doctrine, qui tend à affaiblir la confiance dans l'institution ecclésiastique et à favoriser les mouvements spirituels indépendants, fut particulièrement importante notamment dans l'émergence de l'idéalisme allemand. Jusqu'à aujourd'hui, elle demeure une référence incontournable bien que le plus souvent inconsciente - pour nombre d'associations et de discours ésotériques ${ }^{13}$.

Par ailleurs, dans la première moitié du $\mathrm{XX}^{\mathrm{e}}$ siècle et en particulier sous l'influence des représentants de la " pensée Traditionnelle $»^{14}$, la conception de l'histoire vue comme une succession de cycles fut ravivée par l'apport de la pensée hindoue et connut sous cette forme une certaine fortune en Occident. Dans la bibliographie de Gnôsis, pourtant assez 
laconique, Mouravieff ${ }^{15}$ cite notamment les œuvres de René Guénon, de Léo Schaya, de Titus Burckhardt et de Frithjof Schuon, ce qui atteste sans doute d'une bonne connaissance de ce courant de pensée et, partant, de la doctrine des " cycles cosmiques » qui y est un thème récurrent. Bien que témoignant de conceptions du temps et de l'histoire sensiblement différentes, tous s'accordent sur l'idée que le vingtième siècle constitue la fin d'un cycle décisif dans l'histoire de l'humanité. Comme les penseurs de l'École Traditionnelle, Mouravieff pense que le travail de renouvellement spirituel de l'homme est particulièrement important dans ce moment critique qu'il appelle « Période de transition ", où les dangers qui menacent l'homme, le monde et leur avenir sont très grands. Citant la seconde épître de Pierre, Mouravieff annonce que ce temps pourrait être celui d'une « catastrophe générale $»^{16}$ :

En ce jour les cieux passeront avec fracas, les éléments embrasés se dissoudront, et

la terre, avec les œuvres qu'elle renferme sera consumée. ${ }^{17}$

17 Mais cette période de crise, qui est en fait la Modernité, est en même temps porteuse de grands espoirs puisqu'elle pourrait conduire l'homme à des degrés de conscience jamais atteints auparavant: "l'ère du Saint-Esprit» pourrait ainsi également faire place, toujours selon la prophétie de Pierre, à « de nouveaux cieux et une nouvelle terre où la vérité habitera $»^{18}$. Cela signifie premièrement, pour Mouravieff, que l'homme sera affranchi du travail et de la lutte pour l'existence: «Du simple fait de sa naissance, assure-t-il, l'homme sera doté de tout ce dont il aura besoin pour vivre. Le luxe sera gratuit $»^{19}$. En plus de nouvelles conditions matérielles, l'homme, du moins l'homme d'élite, sera porteur de dons spirituels, et en particulier celui de discerner naturellement le vrai du faux, la vérité du mensonge ${ }^{20}$, ce qui signifie qu'il sera porteur de la vraie Connaissance.

\section{L'ésotérisme, une connaissance cachée}

En effet, pour Mouravieff, «la logique de l'Histoire réclame un esprit nouveau ${ }^{21}$ et cet esprit nouveau, il l'assimile à la « Gnose ", la vraie Connaissance qui doit succéder à la Foi, selon le mot de saint Paul.

L'idée qu'il existe, à l'intérieur de la tradition chrétienne exotérique, une tradition gnostique, ésotérique, révélée par Jésus directement à ses Apôtres, puis transmise par l'intermédiaire de certains pères de l'Église à leurs successeurs, est un lieu commun de la littérature gnostique que notre auteur fait pleinement sien. Ainsi nous retrouvons cette idée déjà répandue à l'époque patristique, notamment chez Clément d'Alexandrie (c.150 c.220). Auteur des Stromates, maître de la Didascalée d'Alexandrie ${ }^{22}$, célèbre pour son exégèse allégorique des Écritures, penseur à la charnière de nombreuses cultures (notamment des cultures judéo-chrétienne et grecque, bien entendu), grand mystique, Clément est avec Irénée de Lyon le père du gnosticisme " orthodoxe » au sein de l'Église. Défenseur d'une vision résolument ésotérique de la doctrine chrétienne, son œuvre inspira beaucoup Mouravieff qui le cite à plusieurs reprises. C'est d'ailleurs en suivant son exemple qu'il rédige lui aussi des Stromates, sous titrées Recueil de notes sur l'enseignement chrétien ésotérique.

Selon Clément, les «traditions secrètes" ou "mystères » compilés dans ses propres Stromates furent transmis jusqu'à lui par une lignée de maitres spirituels, comme il l'explique : 
Ces maîtres, qui conservent la vraie tradition du bienheureux enseignement, issu tout droit des saints Apôtres Pierre, Jacques, Jean et Paul, transmis de père en fils mais peu de fils sont à l'image du père - sont arrivés jusqu'à nous, grâce à Dieu, pour déposer en nous ces belles semences de leurs ancêtres et des Apôtres (...). Le Seigneur a consenti à faire part des divins mystères de cette sainte lumière à "ceux qui pouvaient comprendre" [Mt., 19, 11]. Ainsi donc, ce n'est pas au grand nombre que le Christ a révélé ce qui n'était pas à la portée du grand nombre, mais simplement à une minorité qu'il savait adaptée, capable de recevoir la parole et d'être façonnée selon elle. ${ }^{23}$

21 Sans aucun doute, cet enseignement ésotérique était à l'origine exclusivement oral, et c'est par la tradition orale qu'il s'est transmis jusqu'à Clément. Ce fait n'empêche pas le didascale alexandrin de coucher sur papier les $«$ notes gnostiques ${ }^{24}$ que sont les Stromates, permettant ainsi au lecteur de progresser par lui-même dans la connaissance du mystère de la foi et des Écritures, et donnant par là même à la tradition écrite le statut particulier de transmetteur - aussi partiel et imparfait soit-il - de la tradition orale. De ce fait, tout en veillant à conserver son statut supérieur à l'enseignement oral (qu'il dispensait lui-même à ses catéchumènes), Clément n'exclut pas la possibilité pour la doctrine ésotérique d'être transmise - partiellement - par des écrits. Boris Mouravieff explique ce principe de la façon suivante :

L'hermétisation de la science ésotérique a été pratiquée de tout temps. Le Pentateuque et l'Évangile en sont un témoignage. Mais, tout en cachant le sens exact de la Doctrine, les Anciens prenaient soin de livrer aux profanes - sous une forme ou sous une autre - une part du vrai qui pouvait apparaitre comme un schéma complet. C'est ainsi qu'à travers les siècles, ou même les millénaires, à travers les civilisations éteintes, ils donnaient aux chercheurs de l'avenir les indications suffisantes pour les inciter à des investigations plus approfondies. ${ }^{25}$

Quelque dix sept siècles après clément, notre auteur entend donc s'inscrire dans la même démarche, révélant par ses écrits et par son enseignement la «tradition secrète » des Anciens: non pas une simple connaissance livresque, un savoir profane, mais la Connaissance vivifiante, «supérieure à la Raison et à la Foi ${ }^{26}$. Mais contrairement à ce qu'affirmait son précurseur alexandrin, pour Mouravieff la Gnose divine n'est pas advenue avec le Christ. En effet, la Gnose avait déjà été précédemment transmise à l'humanité au cours du Cycle du Père (l'humanité adamique), mais sous forme de mystères: Mystères de Promesse. Ceux-ci ne trouvèrent toutefois leur entière justification qu'avec l'Avènement de Jésus-Christ, manifestation vivante et réalisation de la promesse : Mystère de Réalisation. La Gnose et les initiés qui en étaient dépositaires furent, par cet événement capital, libérés de la consigne de silence qui pesait sur eux : un foisonnement de théories, d'enseignements et de systèmes put voir le jour, fondés à la fois sur les Mystères de la Promesse et sur le Mystère de Réalisation en Jésus, et qui tentaient principalement d'expliquer la raison de l'imperfection du Monde et de la présence du Mal. Il résulta de ces multiples spéculations deux courants majoritaires, que Mouravieff oppose de la façon suivante: une gnose hétérodoxe, qui attribuait cette imperfection à une catastrophe cosmique ou à la malveillance du Créateur, et une gnose orthodoxe, qui axait son enseignement sur la perfectibilité de l'homme qui marche à la suite de Jésus et sur la possible divinisation de son humanité par le moyen de la "deuxième naissance ». C'est, selon notre auteur, cette seconde - et seule légitime -Gnose qui survécut, grâce à l'œuvre des Apôtres et des grands docteurs œcuméniques de l'Église et en particulier de l'orthodoxie orientale. Mouravieff pense que par ce fait, la tradition ésotérique elle aussi put être préservée dans sa pureté originelle, soit dans les temps anciens à l'abri de 
l'hermétisme, dans les monastères grecs et russes notamment, ainsi que dans les écrits philocaliques $^{27}$; soit dans le temps présent de façon dévoilée, dans sa propre œuvre littéraire (en particulier dans Gnôsis qui en est l'exposé complet) et spirituelle (les Stromates et la pratique enseignée dans le C.E.C.E.). Pour notre auteur, en effet,

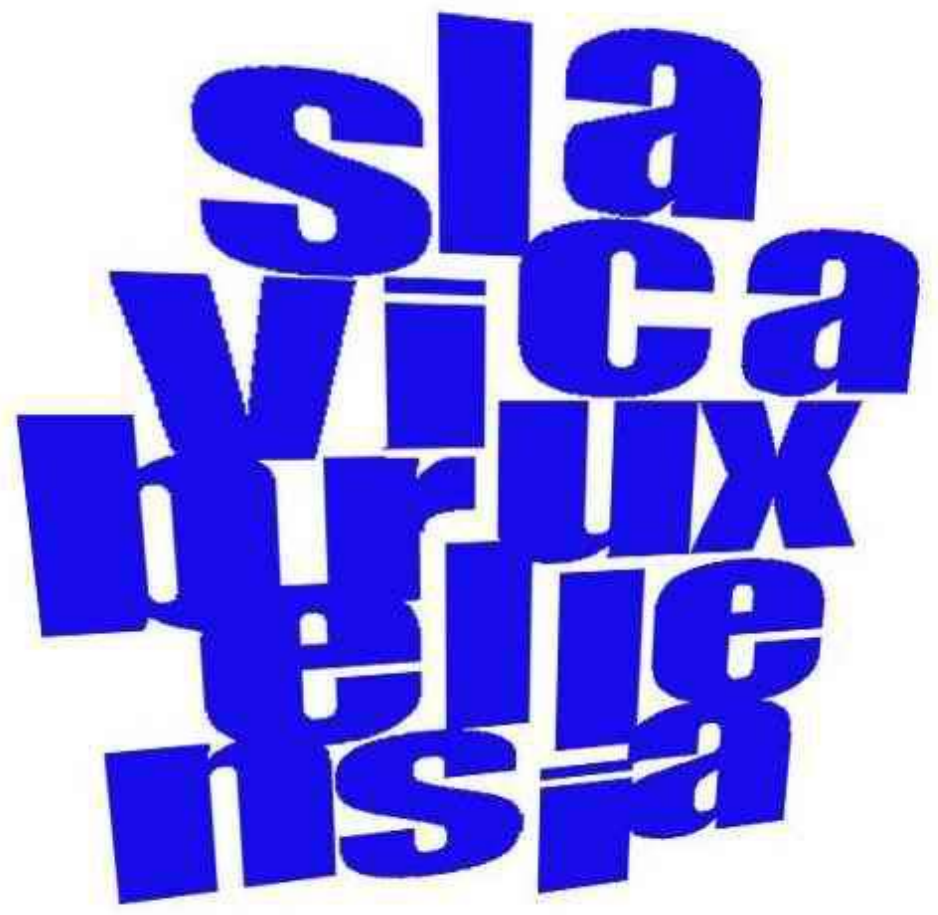

GNÔSIS est la première divulgation de l'ensemble, en abrégé, mais laissant au chercheur persévérant la possibilité d'aller en profondeur, de la Gnôse communiquée par Jésus, après Sa Résurrection, à Pierre, Jean et Jacques, et qui constitue le contenu de la Tradition Chrétienne ésotérique. ${ }^{28}$

C'est donc aux «racines", à la "source $»^{29}$ de la Tradition ésotérique chrétienne que Mouravieff entend puiser afin de présenter la doctrine dans son intégralité : les textes sacrés de l'Ancien et du Nouveau Testament, les commentaires dont ils font l'objet (commentaires patristiques et en particulier ceux réunis dans la Philocalie), ainsi que l'enseignement et la discipline reçue par les personnes régulièrement investies dans leur fonction de maîtres spirituels (la "Grande Confrérie Ésotérique ») ${ }^{30}$.

À côté de ces enseignements écrits, il est nécessaire qu'il y ait également un enseignement oral afin de vivifier la lettre, de passer des simples croyances, articles de foi, dogmes et credo (sans nier leur valeur pédagogique et relative), à une connaissance plus parfaite, une véritable seconde naissance, qui ouvre les yeux de l'intelligence « au vrai sens de la vie, [...à] la signification de la mission du chrétien dans l'Ère Nouvelle, [à] la possibilité [qui] est offerte de s'initier à cette Sagesse divine, mystérieuse et cachée »" Mais, il faut le souligner, dans l'esprit de Mouravieff, il ne suffit pas de saisir en quelque sorte mentalement la doctrine exposée dans Gnôsis pour parvenir à cet état de plénitude spirituelle. Gnôsis n'est que l'exposé de la doctrine : elle n'est ni son application, ni son interprétation, ni sa compréhension plénière. Aux élèves expérimentés du Centre d'Études Chrétiennes Ésotériques, qui sont par le fait même des lecteurs attentifs de Gnôsis, Mouravieff adresse des Stromates, aussi appelées L'Art de Vaincre, dans l'introduction desquelles il rappelle que : 
La lecture spontanée de "Gnôsis", même si elle est attentive, répétée, faite avec l'assiduité voulue, ne peut conduire qu'au Savoir de la Doctrine. Le lecteur sait que pour parvenir au niveau du Comprendre, il faut ajouter au Savoir quelque chose d'impondérable, un epsilon. Les préceptes et les exercices recommandés dans les Stromates ont pour but d'aider les étudiants à gagner cet epsilon. Il leur ouvrira les chemins du Comprendre qui, à son tour, les conduira vers le Savoir-faire. ${ }^{32}$ favorables. C'est la raison pour laquelle en de nombreux endroits Mouravieff indique que l'ésotérisme n'est pas une voie qui convient à tout le monde, mais qu'elle est au contraire réservée à une élite : car si l'enseignement est transmissible dans ses formes, donc du point de vue extérieur, en réalité l'acquisition de la connaissance initiatique ne peut être le fait que du travail personnel de chaque étudiant. Ce travail comprend notamment un aspect de prière, dont il n'est pas inutile de dire ici quelques mots.

\section{La tradition hésychaste}

L'enseignement hésychaste est au centre de la pratique orthodoxe de la prière, et ce depuis les origines chrétiennes. Le terme «hésychasme» (du grec hésychia, la "quiétude » au sens de "calme intérieur ", de " paix de l'âme ») désigne une méthode spirituelle connue dans tout l'orient chrétien et en particulier dans les monastères d'Egypte ainsi que, plus tard, dans les monastères du mont Athos, mais aussi en occident où, par l'intermédiaire de Jean Cassien, elle fut connue des premiers moines bénédictins. Les Pères "neptiques» (du grec nepsis, «sobriété », "vigilance ", «lucidité »), parmi lesquels figurent Évagre le Pontique, Maxime le Confesseur ou Jean Climaque, qui sont à l'origine de cette tradition, apprennent à rechercher et à maintenir un profond silence de l'âme et des passions par la prière ininterrompue et le souvenir permanent de Dieu, et à garder le cœur en état de recevoir la présence de l'Esprit. Depuis le XIVe siècle, avec la victoire de saint Grégoire Palamas (1296 - 1359) - qui pousse l'oraison hésychaste jusque dans ses conséquences ultimes avec la doctrine de la déification de la personne par sa participation aux énergies divines - contre les adversaires de cette pratique spirituelle et de la théologie qui l'accompagne, l'hésychasme devient en quelque sorte la «doctrine officielle » de l'Église orthodoxe.

La publication de la Philocalie des pères neptiques viendra renforcer ce mouvement ${ }^{33}$. Ce livre, qui est une sorte d'anthologie de textes portant sur la prière hésychaste, verra le jour en grec à la fin du XVIII siècle et sera, à travers sa traduction slavonne ${ }^{34}$, à l'origine $\mathrm{du}$ renouveau spirituel russe du XIX ${ }^{\mathrm{e}}$ siècle dont les Récits d'un pèlerin russe ${ }^{35}$ sont l'exemple littéraire le plus célèbre. Comme l'écrit Elisabeth Behr-Siegel, la Philocalie « fut, pendant la première moitié du XIX siècle [...] la nourriture spirituelle préférée des moines russes $»^{36}$. Il n'y a donc rien d'étonnant à ce que Boris Mouravieff le considère comme une source spirituelle de premier ordre.

Conformément à la tradition hésychaste, Mouravieff enseigne une technique de prière, en apparence simple, qui consiste à répéter inlassablement, jusqu'à dix ou vingt mille fois par jour, l'invocation suivante: "Seigneur Jésus-Christ, Fils de Dieu, aie pitié de moi, pécheur ". Cette prière porte le nom de " prière de Jésus ", car sa puissance provient de l'invocation du nom divin, ou de "prière du cœur", car elle se fait depuis la tête en direction du cœur considéré comme le centre de la personne et le réceptacle privilégié de la présence divine ${ }^{37}$. 
Les exercices spirituels, qui s'adressent nécessairement aux étudiants possédant un certain bagage théorique, ne s'arrêtent toutefois pas là. Sans entrer dans de nombreux détails qui risqueraient d'alourdir notre exposé, notons que Mouravieff leur assigne globalement trois objectifs, en conformité avec la structure tripartite de l'être humain : corps, âme, esprit. Ces objectifs sont la maîtrise du corps (le Moi corporel), la maîtrise de la Personnalité (le Moi personnel), et la prise de contact avec les niveaux supérieurs de la conscience (le Moi réel) ${ }^{38}$. Il s'agit là d'une reprise de la classique tripartition établie par les stoïciens et reprise par les pères de l'Église : practikè, gnosis phusikè, theologikè.

\section{La fin de l'ésotérisme: l'Amour}

Si l'enseignement est intégré, vécu et réalisé jusqu'au bout, grâce à la pratique et à l'étude de la doctrine, alors selon Mouravieff l'étudiant est en mesure de passer de la Foi à l'Espérance puis, au-delà de celle-ci, à la Connaissance ou Gnose :

Ainsi, sans la Foi au cœur, il est impossible d'atteindre, au sens ésotérique, l'Espérance. Et sans l'une ni l'autre, on ne parvient jamais à Gnôsis, la connaissance vivante [...]. Le langage imagé de la Tradition appelle la triade: Foi, Espérance, Connaissance : l'Epée à triple tranchant, ou encore la Lame triangulaire. ${ }^{39}$

31 Mais cette triade ne réalise pas encore à elle seule la seconde Naissance promise à l'homme d'élite. En effet, il ne s'agit là que d'étapes, certes nécessaires, mais non suffisantes, car le but ultime de toute réalisation spirituelle est l'Amour. Seul l'Amour, comme l'enseigne Mouravieff après saint Paul, est un état non plus partiel et transitoire, mais définitif. Pour lui, la Foi et la Connaissance seront amenées à disparaître lorsque sera accompli ce qui est parfait :

Or le parfait, c'est l'Amour qui comprend en lui l'accomplissement de toutes les vertus, de toutes les prophéties, de tous les mystères et de toute Connaissance [cf. I Co., 13] $]^{40}$

Il convient d'insister encore un instant sur ce point, car par celui-ci Mouravieff se distingue de la plupart des auteurs "gnostiques " (qui s'arrêtent généralement à la question de la "connaissance»), tout en se rapprochant d'un discours qu'on dirait volontiers « mystique ». Il se conforme en cela à la Philocalie et, plus généralement, à toute pensée vraiment chrétienne, qui fait nécessairement de l'Amour le terme de l'économie du salut. Pour résumer ce qui a été dit sur ce sujet, et pour rappeler la vision cyclique de l'histoire précédemment exposée, citons encore Mouravieff :

La face du monde change. Le Cycle du Fils, comme jadis celui du Père, arrive à son terme. Avec le Christ, la Loi reçue par Moïse prit fin (Rom. 10,4) et fut remplacée par le régime de la Foi, de l'Espérance et de l'Amour (I Co. 13, 13). À présent, avec les guerres et les révolutions du siècle, avec les progrès extraordinaires de la science positive, nous sommes entrés dans la période transitoire dont la signification est d'ouvrir l'accès au Cycle du Saint-Esprit. Au cours de cette période, la Foi sera progressivement remplacée par la Connaissance et l'Espérance sera abolie dans l'Accomplissement. Ce sera le triomphe final de l'Amour. ${ }^{41}$

Cette victoire de l'Amour, qui épargnera au genre humain la "catastrophe générale» prophétisée par saint Pierre, devrait assurer la venue de l'Ère du Saint-Esprit et, par lui, apporter sur Terre la Lumière, la Vérité et la Vie ${ }^{42}$. C'est du moins l'espérance de Boris Mouravieff, et la phrase sur laquelle il conclut son cycle des trois volumes de Gnôsis, soit l'exposé théorique de la « Tradition ésotérique ». 


\section{L'enseignement pratique de la " gnose » : le C.E.C.E.}

L'aspect pratique, concret, de l'enseignement gnostique de Boris Mouravieff était assuré par le Centre d'Études Chrétiennes Ésotériques ainsi que par les groupes de travail qu'il supervisait. Ces groupes devaient être composés d'un maximum de douze membres nombre dont la symbolique apostolique est certainement voulue - se réunissant une fois par semaine pour traiter d'un aspect particulier de la doctrine. Le principe de fonctionnement voulait que chaque membre choisisse un passage de Gnôsis à propos duquel il méditait et préparait un exposé d'une trentaine de minutes. Il s'ensuivait une discussion à laquelle chacun devait prendre part. Conformément à l'ordre que Mouravieff a voulu donner à son œuvre, les sujets devaient être choisis d'abord dans Gnôsis I : cycle exotérique, en suivant l'ordre des chapitres, puis dans Gnôsis II : cycle mésotérique, avant d'atteindre le volume de Gnôsis III : cycle ésotérique. Quelques réunions extraordinaires, au cours desquelles des membres du Comité directeur du C.E.C.E. ou des conférenciers extérieurs étaient reçus, devaient également animer la vie des groupes de recherche ${ }^{43}$.

Le fonctionnement interne du Centre et des groupes de travail, la fréquence et la procédure de leurs réunions, témoignent d'une certaine ambition de la part de Mouravieff. Il s'y consacre d'ailleurs pleinement à partir de 1962, après avoir quitté l'Université de Genève. Plusieurs branches secondaires du C.E.C.E. seront créées dans un certain nombre de villes, notamment à Paris, à Lille, à Bruxelles, et même au Caire et au Congo ${ }^{44}$. Le maitre leur adresse régulièrement des «Bulletins d'information $»^{45}$, ainsi qu'occasionnellement des conseils spirituels réunis sous le nom de Stromates. Hélas, quelques années plus tard, suite à de graves problèmes de santé, il meurt à Genève d'une crise cardiaque, le 28 septembre 1966. Le C.E.C.E., et a fortiori les groupes d'étude constitués sous sa direction, cessent prématurément leurs activités. Sa veuve, Larisa Mouravieff se chargera de faire paraître plusieurs chapitres inédits des Stromates et de veiller sur les archives du Centre, qu'elle dépose finalement à la Bibliothèque Publique et Universitaire de Genève où un Fonds Boris Mouravieff est destiné à l'usage des chercheurs intéressés.

Depuis le mois de mai 2000, l'Association Boris Mouravieff, créée à Paris, s'est fixée pour but principal de veiller à la continuité de la diffusion de l'œuvre ésotérique de Boris Mouravieff, notamment en rééditant les trois tomes de Gnôsis, ainsi qu'en réunissant en un volume divers articles qui étaient devenus introuvables ${ }^{46}$.

\section{Quelques conclusions}

Depuis sa jeunesse, mis sur la voie par son grand oncle ainsi que par diverses rencontres (notamment celles d'Uspenskij et de Gjurdjžiev), Boris Mouravieff s'est intéressé de près à l'ésotérisme et à la Tradition orthodoxe. À la fois dans ses cours à l'Université de Genève, dans ses volumes de Gnôsis et au sein du C.E.C.E., il enseigne une doctrine qu'il estime être la «tradition ésotérique». Cette Tradition, universelle dans son essence, aurait été révélée à l'humanité sous divers aspects, mais c'est sous sa formulation chrétienne qu'elle est véritablement parvenue à son niveau définitif, car le christianisme est lui-même universel. La doctrine aurait été enseignée par Jésus à ses Apôtres, puis par eux aux saints pères de l'Église: elle est donc, comme le dit Mouravieff, "fondamentalement chrétienne ». Il en expose les divers aspects en s'appuyant sur les textes bibliques vétéro- 
et néo-testamentaires, sur les commentaires patristiques compilés dans la Philocalie, ainsi que sur une série d'exercices spirituels conformes à la tradition hésychaste. L'enseignement de Boris Mouravieff, résolument initiatique, comprenait donc des aspects théoriques (étude de la doctrine) et pratiques (exercices spirituels et réalisation de l'enseignement). Le but de cet enseignement est de faire parvenir l'étudiant à des degrés de conscience supérieurs et, finalement, à la réalisation plénière de sa Personnalité, par la «seconde Naissance » qui assure l'entrée dans le Royaume de Dieu. L'étudiant, devenu homme d'élite, est alors chargé de préparer l'humanité à une phase cruciale de l'histoire : la « Période de transition » qui marque l'entrée dans le «Cycle du Saint-Esprit».

\section{NOTES}

1. Sur ces questions, voir Mouravieff B., Gnôsis, Neuchâtel (Suisse), Éd. de La Baconnière, 1969, t. 1, pp. 13 et 14 .

2. Voir Faivre A., "Boris Mouravieff ", in: Dictionary of Gnosis \& Western Esotericism, Leiden (Pays-Bas), Londres, Brill, 2005, t. 1, pp. 806-807.

3. Saint-Pétersbourg, 1838. Le livre a été traduit en anglais par le Révérend R. W. Blackmore, Londres, éd. John Henry Parker, 1842 (rééd. Montana, Éd. Kessinger Publishing Co, 2004).

4. Les quelques éléments biographiques sont tirés de la préface à Mouravieff B., Écrits sur Ouspensky, Gurdjieff, et sur la Tradition ésotérique chrétienne, Dervy, Paris, 2008, pp. 7-28, et du site de l'Association Boris Mouravieff, «Éléments bibliographiques », http://www.association-borismouravieff.com/index.html

5. Paris, Stock, 1950 [1961].

6. Dans son article «Ouspensky, Gurdjieff et les Fragments d'un enseignement inconnu » (1957), in : Écrits...Op.cit., pp. 95-138, Mouravieff explique les points qu'il juge problématiques dans la méthode d'enseignement de Gjurdžiev et dans le « système » d'Uspenskij.

7. Neuchâtel (Suisse), Éd. de La Baconnière, 1949.

8. Paris, Payot, 1962.

9. Pour une bibliographie complète, voir Mouravieff B., Écrits..., Op. cit., pp. 341-342.

10. Eph. 4, 22-24.

11. En réalité Mouravieff dénombre tantôt trois, tantôt cinq phases, selon le point de vue qu'il choisit. Les cinq phases sont (avec leur élément déclencheur) : 1. Cycle préhistorique (chute d'Adam), 2. Cycle du Père (Déluge des Eaux), 3. Cycle du Fils (Destruction du Temple), 4. Cycle du Saint-Esprit (Hiroshima), 5. Mille ans sans guerre = Millénium (l'Accomplissement $:$ soit le Déluge du Feu, soit de Nouveaux Cieux et une Nouvelle Terre). Voir Mouravieff B., Gnôsis, Op. cit., t. 2, p. 74.

12. Ibid, t. 1, p. 19.

13. Sur ce point on consultera de Lubac H., La Postérité spirituelle de Joachim de Flore. 2 vol, Lethielleux, Paris, 1981.

14. Par cette expression nous désignons l'école de pensée qui s'est formée dans le sillage et la continuation de l'œuvre du philosophe français René Guénon. Appelée "École Traditionnelle » par ses représentants, elle prend le nom de «Perennialism» dans le monde anglo-saxon en référence à la Philosophia Perennis dont ils se veulent les héritiers et continuateurs. Pour l'histoire 
de ce mouvement on lira, récemment traduit en français : Sedgwick M. J., Contre le monde moderne. Le traditionalisme et l'histoire intellectuelle secrète du XX $X^{e}$ siècle, Dervy, Paris, 2008 [ $1^{\mathrm{e}}$ éd. : 2004].

15. Il s'agit là d'une hypothèse. En effet, la bibliographie a été « préparée avec la collaboration du Docteur Albert-Jean Lucas ", et il est difficile de connaître l'exacte mesure dans laquelle celle-ci reflète les sources authentiques de Mouravieff.

16. On lira quelques-unes des considérations sur ce point dans son article «Le problème de l'Homme nouveau », in : Écrits..., Op. cit., pp. 29-43.

17. 2 P., 3, 10-11

18. 2 P., 3, 13. Nous citons d'après la traduction de l'article cité en note 5.

19. Mouravieff B., «Le problème de l'Homme nouveau ", Art. cit., p. 29.

20. Ibid., p. 39.

21. Voir Mouravieff B., Gnôsis, Op. cit., t. 1., p. 18.

22. La "Didascalée » ( $\mathrm{du}$ grec $\delta i \delta \alpha \sigma \kappa \alpha \lambda \tilde{\varepsilon i o v}$, "école ») était une célèbre école catéchétique et théologique où furent formés quelques-uns des plus grands théologiens et Pères de l'Église (notamment Origène, Basile le Grand, Grégoire le Thaumaturge, Grégoire de Naziance). Selon la Tradition, elle fut fondée, par saint Marc, mais plus vraisemblablement par Pantène au II e siècle, didascale (« maître ») auquel succédèrent Clément et Origène.

23. Mouravieff B., Stromates I, Éd. du Cerf, coll. « Sources Chrétiennes », Paris, 1951, p. 52.

24. Le titre complet de son grand ouvrage est Stromates de notes gnostiques selon la vraie philosophie. 25. Mouravieff B., Gnôsis, t. 1, Op. cit., pp. 129-130. Il faut noter à cet endroit que ce principe tel qu'expliqué par Mouravieff ressemble de très près à ce que les penseurs de l'École Traditionnelle nomment «Tradition primordiale ». Sur ce point précis, on lira avec intérêt l'article d'Antoine Faivre: "Histoire de la notion moderne de Tradition dans ses rapports avec les courants ésotériques (XV'-XX siècles) ", in : Symboles et Mythes dans les mouvements initiatiques et ésotériques (XVII $-X X^{e}$ siècles) : Filiations et emprunts, Archè - La Table d'Émeraude (revue «ARIES », hors série), Milan-Paris, 1998, pp. 7-48.

26. Mouravieff B., Gnôsis, Op. cit., t. 1, p. 287.

27. La tradition orthodoxe appelle "philocalique» (qui signifie littéralement "amour de la beauté ») tout texte spirituel, mais ce vocable s'applique avant tout à l'anthologie réalisée par Nicodème l'Hagiorite et Macaire de Corinthe en 1782, aujourd'hui appelée Philocalie des Pères Neptiques ou, plus simplement, la Philocalie. Mouravieff y renvoie en de nombreux endroits de son œuvre. En dehors des éditions complètes, il en existe en français une version abrégée et facilement accessible: Grouillard J., Petite Philocalie de la prière du cour, Seuil, coll. "Points/ Sagesse », Paris, 1979.

28. Cité in : Mouravieff B., Écrits... Op. cit., p. 8.

29. Mouravieff B., Gnôsis, Op. cit., t. 1, p. 10.

30. Voir Idem.

31. I Co.,2, 6. Voir Ibid., pp. 14-15.

32. Introduction à «L'Art de Vaincre. Les Stromates. Recueil de Notes sur l'enseignement chrétien ésotérique ", in : Mouravieff B., Écrits..., Op. cit., p. 261.

33. Sur la réception de la Philocalie dans l'Église orientale, il est indispensable de lire Deseille P., La spiritualité orthodoxe et la philocalie, Albin Michel, coll. "Spiritualités vivantes », Paris, 2003 [1997].

34. La Dobrotoljubie fut publiée à Saint-Pétersbourg en 1794 par les soins de Paissij Veličkovskij.

35. Publiés anonymement et pour la première fois à Kazan en 1865. La traduction française des quatre premiers récits fut éditée en 1943 aux Éd. de la Baconnière. Sur le pèlerin russe et sur ce genre littéraire, voir Evdokimov M., Pèlerins russes et vagabonds mystiques, Éd. Du Cerf, coll. « Patrimoines », Paris, 2004, pp. 145-192, passim.

36. Behr-Siegel E., «La prière à Jésus ", in : Dieu vivant, $n^{\circ} 8$, p. 71, cité in : Grouillard J., Op. cit., p. 12. 
37. Pour une clarification de ces notions, on lira avec profit Serr J \& Clément O., La prière du cœur, Éd. De l'Abbaye de Bellefontaine, Bégrolle (France), 1977.

38. Voir Mouravieff B, Gnôsis, Op. cit., t. 1, pp. 233 et sq.

39. Ibid., t. 2, p. 228.

40. Ibid., t. 1, pp. 1819 .

41. Ibid., t. 1, p. 154.

42. Ibid., t. 3, p. 260.

43. Le règlement du C.E.C.E. et des " groupes de travail » se trouve en annexe du troisième tome de Gnôsis, Op. cit., pp. 261-264.

44. Association Boris Mouravieff, «Eléments bibliographiques », http://www.association-borismouravieff.com/index.html

45. Plusieurs de ces bulletins sont édités dans Mouravieff B., Écrits..., Op. cit., pp. 313-340.

46. C'est ce volume qui constitue les Écrits sur Ouspensky..., Op. cit.

INDEX

Index géographique : Russie

Index chronologique : entre-deux-guerres, communisme

Mots-clés : ésotérisme chrétien, orthodoxie

\section{AUTEURS}

\section{OLIVIER SANTAMARIA}

Doctorant à l'Université Libre de Bruxelles (Belgique), aspirant FNRS ; membre du Centre interdisciplinaire d'étude des religions et de la laïcité (CIERL) de l'Université Libre de Bruxelles (Belgique) 